\title{
THE POTENTIAL OF WEARABLE DEMONSTRATORS INTRODUCING INNOVATIVE TECHNOLOGIES
}

\author{
L. -M. Lüneburg ${ }^{凶}$, E. Papp and J. Krzywinski \\ Technische Universität Dresden, Germany \\ $\square$ lisa-marie.lueneburg@tu-dresden.de
}

\begin{abstract}
With the introduction of ' $5 \mathrm{G}$ ' data transfer gets faster and further reaching than ever before. This new communication technology paves the way for an exchange of skills and competencies between humans and machines. This raises the question of how future users can profit and understand the potential brought about by these technologies. This paper elaborates the use of demonstrators in a pilot study as research tools and assesses their potential. It gives first insights why demonstrators are suitable to set a basis for public recognition for body-worn CPS and how to promote innovative visions.
\end{abstract}

Keywords: tactile internet, user-centred design, wearables, internet of things (IoT), cyber-physical systems

\section{Introduction}

With the introduction of the fifth mobile communication standard, ' $5 \mathrm{G}$ ' data transfer gets faster and further reaching than ever before. It allows the broadcast of large amounts of data over long distances and in near real-time. This new communication technology paves the way for an exchange of skills and competencies between humans and machines that is neither time- nor location-bound. As a consequence, long-distance haptic feedback (Tactile Internet) and new ways of spatially distributed cooperation and learning (Internet of Skills and Competencies) will no longer be a concept of the future. This is where cluster of excellence 'CeTI' engages with extensive research that makes for a variety of interactive use cases. Conceivable areas of application could include the consumer or professional sector. Within an interdisciplinary pilot study, designers, engineers and computer scientists identified feasible application scenarios and implemented physical demonstrators to make the Tactile Internet with Human-in-the-Loop (TaHil) come alive. As one of the application areas, competitive sports have been emphasised for their broad level of acceptance. Requiring exceptional discipline, high physical strain and sequences of perfected motion, professional sports have a great potential for the development of efficient training methods with the aid of cyber-physical systems. In the scope of this pilot study, another key element was to examine to what extent demonstrators are able to provide an understanding of an abstract technology which still remains in a very early state of scientific research and development. Therefore, factors for a meaningful technology application were identified form literature. The study showed that these factors are valuable to classify demonstrator characteristics which promote the communication of a novel technology. 


\section{Demonstrators as translator objects for TaHil applications}

The Tactile Internet with Human-in-the-Loop (TaHil) paves the way for significant breakthroughs in enabling humans to interact with co-operating Cyber-Physical Systems (CPS). Based on intelligent wide-area-communication networks, TaHil promotes equitable access to remote work and learning environments for people of different genders, ages, cultural backgrounds, or physical capabilities.

Within this project, the "Internet of Skills" explores how to democratise the access to competencies and expertise for everyone through co-operating CPS. TaHil devices will be developed to allow the training of relevant skills to learners, e.g. in specified individual training activities supervised by a remote human teacher in quasi real-time, or pushing the concept further, the machine itself becomes an unsupervised teacher. Such devices could be wearables as well as soft exoskeletons containing fast sensors and actuators with multimodal haptic feedback for human perception and action. Along with electronics that precisely localise humans and objects in real-time, TaHil devices will able to promote learning of motoric skills. However, an understanding of TaHil's possibilities remains difficult and vague. Moreover, as these systems are worn directly on the human body, it is indispensable to create a high level of trust and approval within the society.

Therefore, three demonstrators (two wearables and an exoskeleton) were developed as a tangible means to present possibilities of TaHil technologies, even though most of its technologies still have to be developed in the next seven years. The aim was to enhance understanding as well as acceptance by different target groups through performance use cases with exemplary applications. On this basis, a design process was launched in order to create wearable demonstrators which motivate an open discourse and feedback about the use of those technologies.

The demonstrators were designed in 6 months by three interdisciplinary teams comprised of designers, engineers and computer scientists. Utilizing user centred design methods like open interviews with sport professionals e.g. choreographs and dance students, the teams identified three use cases in the different professional sports areas. Digital and physical rapid prototypes were evaluated together with the sport experts and gave more in-depth insight in the appropriate usage for the TaHil technologies. Futhermore, the design process was oriented according to the following questions:

- Who is the target audience?

- How can the Tactile Internet be experienced through a demonstrator in a positive way?

- How can the credibility of a concept be reinforced through the usage of a demonstrator?

- How realistically can the demonstrator be implemented?

- Which level of technical complexity can be reached?

Subsequently, the demonstrators were created with full haptic feedback and sensoric functions. The goal was to maintain a market-ready product appearance including hardware as well as software. A pilot study was then carried out in order to examine whether the demonstrators were suitable to set a basis for public recognition and acceptance for CPS, and therefore to identify which factors could be essential.

\section{Literature and research gap}

Even though the creation and usage of physical demonstrators in innovation processes and scientific research is acquiring greater importance, regarding the abilities of demonstrators beyond the design respectively product development process is rarely discussed in scientific literature. Moultrie (2015) set a first basis with his approach of demonstrators as 'translator objects' in contrast to 'boundary objects'. These 'translator objects' are results out of innovation and design processes. Both, innovation and design processes, share many similarities and often are linked to each other, which was widely discussed in the past years (Verganti, 2009; Dell'Era et al., 2010; Price and Wrigley, 2016).

\section{Impact and application of demonstrators}

The overall impact of digital demonstrators on interaction interfaces is already described and assessed in various publications (Lauff et al., 2018). In theory, their findings can be translated to physical demonstrators that act as a user interface between human and machine. 
In the development of digital user interfaces, prototypes are used as vehicles for the evolution and demonstration of visions of innovative systems (Bäumer et al., 1996). Prototyping is a developmental approach improving the planning and execution of development projects. It is well suited to gather experiences in new fields of application and thus support the incremental or evolutionary development of product systems.

In general, it is not important that those prototypes depict domain-specific and technical aspects in great detail (Bäumer et al., 1996). However, it is important that they illustrate the intended solution and make it easily comprehensible.

Moreover, the study shows that prototypes are well suited for the exchange of specialist and technological knowledge, and for the evaluation of the resulting insights with a team of experts. It has also been observed that prototypes represent an important means of communication between developers and end-users. As a source of innovation, prototypes are used not only for individual software projects, but also for various ways of marketing research and field studies (Bäumer et al., 1996).

Lauff et al. (2018) defines a prototype as "a physical or digital embodiment of critical elements of the intended design, and an iterative tool to enhance communication, enable learning, and inform decision-making at any point in the design process." (Lauff et al., 2018, p.10)

From the viewpoint of futurologists, Bell et al. (2013) and Kymalainen (2016) describe how sciencefiction prototypes effect technological developments in the present. Innovation and its promotion are often inspired by fictional concepts of the future (Kymalainen, 2016), that put technological novelties in a socio-cultural context and thus raise their credibility and the possibility for a market launch.

Demonstrators are usually viewed as technological prototypes which are close to market (Moultrie, 2015). In his study, Moultrie (2015) describes the extent to which they can contribute to scientific research progress from the laboratory to the marketplace. Therefore, he identifies their ability in enhancing non-linear and agile processes in order to translate from science to market. Based on the 'Science, Technology, Application, Market' (STAM) model, Moultrie (2015) classifies demonstrators created in scientific contexts by referring to the purposes which they might fulfil. Those demonstrators which are able to span boundaries between disciplines as well as those of scientists and their scientific community are called 'translator objects.' In addition, Moultrie (2015) observed in his studies that these objects were also effective in communicating the potential purposes of scientific enquiries.

The question of which characteristics influence the ability of demonstrators to communicate or rather translate the potential of these scientific outcomes still remains open. In literature, the impact of design adding meaningfulness to innovative technologies is described as one crucial determinant for a successful application which can then be named an innovation.

There are a number of definitions for the concept of innovation in scientific literature. Innovations are mostly characterised by their level of novelty (Garcia and Calantone, 2002).

However, current literature assumes that technological and functional advantages over existing products are not really a guarantee for success in innovation (Chiesa and Frattini, 2011). According to the branch of industry, 40 to 90 per cent of all new products are not accepted by consumers (Feiereisen et al., 2008). Pure functional superiority is not enough to convince users of a new product.

The success of innovation is dependent on how it is perceived and assessed by the user (Henard and Szymanski, 2001). This phenomenon, as described from an economics perspective, has been formerly framed by Verganti (2009) and Utterback, who established theories of design-inspired or designdriven innovation. According to Utterback and colleagues (2006), successful innovation can be defined as the right balance between technology, market and significance (as seen in Figure 3). The symbolic and emotional value of a product as well as the socio-economic needs of the user are equivalent to the actual functionality.

Innovations are classified as 'meaningful' by consumers when they are perceived as desirable, useful and convenient (Arts et al., 2011). Consumers tend to strike a balance between perceived profits and losses that result from the integration of an innovation into their daily routine (Tomczak et al., 2016). The St. Gallen Business Innovation Model also assumes that consumers are predominantly concerned with whether and to what extent the available innovations can be integrated into their daily routine. Other points of interest could be the potential to provide new experiences or benefits (Gourville, 2006). 


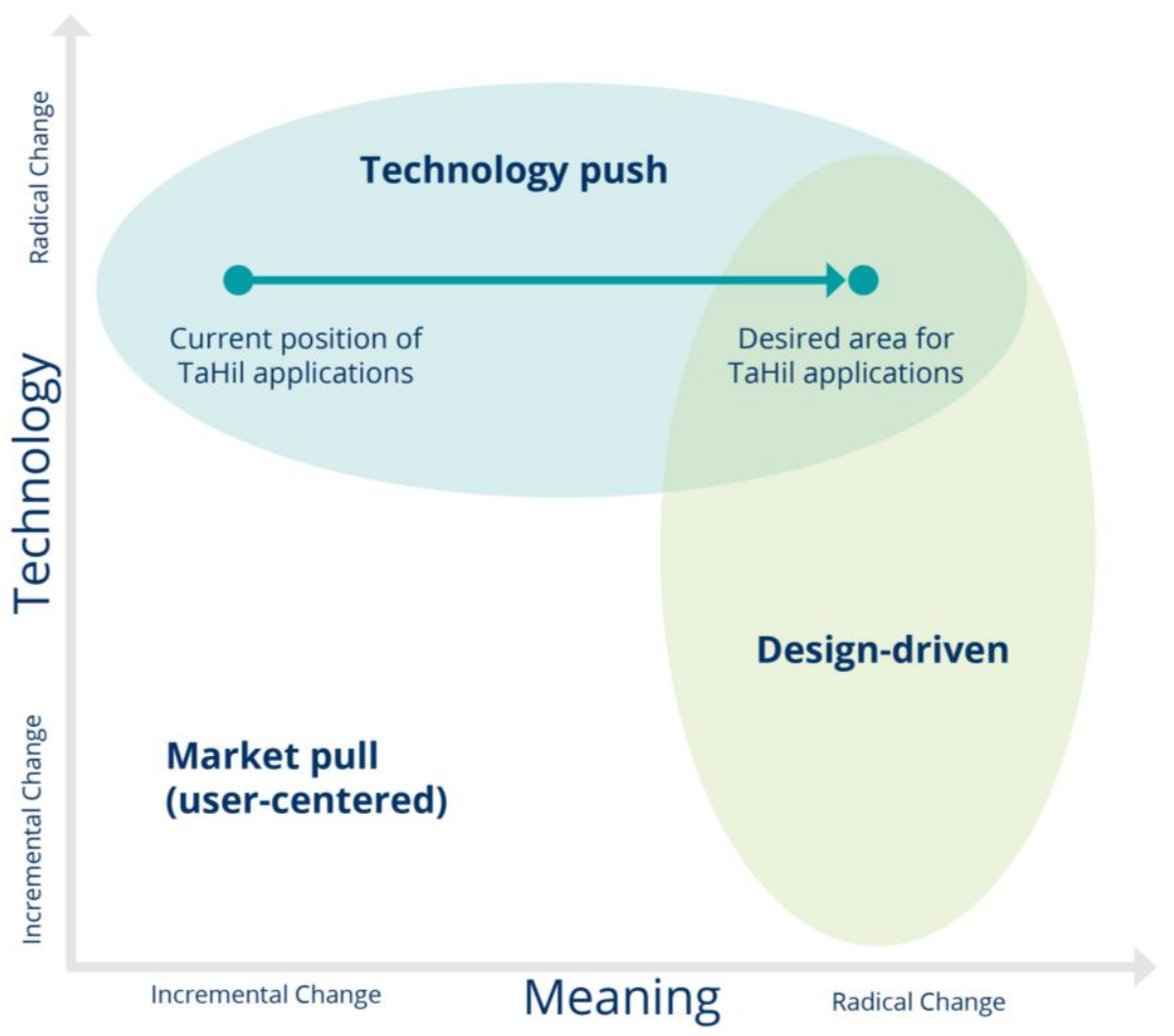

Figure 1. Design-driven innovation (Verganti, 2009)

The perception of meaningfulness is dependent on the functionality and usability of an innovation as well as emotional and psychological considerations. This can be exemplified by the reaction of the social environment regarding the usage of an innovation or the level of joy associated with it (Tomczak et al., 2016). The Unified Theory of Acceptance and Use of Technology (UTAUT) provides a systematic overview on the factors that decisively influence the perceived meaningfulness of an innovation (Venkatesh et al., 2012):

- Performance

- Estimated effort

- Social influence

- Facilitations

- Hedonic motivation

- Value for money

- Habit

These seven factors imply that the assessment of the meaningfulness of an innovation is a multilayered and complex process (Tomczak et al., 2016). Within the context of the St. Gallen Business Innovation Model, the meaningfulness of an innovation is rated as the main criteria for the purchase decision by a customer (Tomczak et al., 2016), whereas the level of novelty is regarded as a means to attract attention.

This leads to following hypothesis: These seven factors of perceived meaningfulness are able to influence the experience of demonstrators and with that, the acceptance of novel technologies at an early stage of research. However, the role of designed demonstrators in progressing technologies from the laboratory into successful innovations is not fully understood. Therefore, the aim is to explore specifically how those factors might be beneficial to manipulate demonstrator characteristics in order to raise acceptance for innovative solutions and how those effects can be assessed.

For this reason, we conducted a pilot study to investigate how to characterise more specifically the influencing factors of a demonstrator's design. Both the demonstrators that were created and the study 
will be introduced in the following section with a particular focus on the public perception of the demonstrated technologies.

\section{Case studies}

In order to be able to demonstrate the potential of innovation associated with $5 \mathrm{G}$, a study project was launched. Within the scope of the project, three case studies exemplified the application context of 'competitive sports.' The aim was to create tangible prototypes that allow for haptically perceptible innovative visions. Especially, how learning can be promoted through vibrotactile, visual or force feedback. The fully functional 5G sports prototypes were then presented at the IEEE 5G Summit, an international symposium in the field of 5G technology.

In this context, a pilot study with three designed demonstrators was carried out while observing factors which influenced whether there were positive or negative experiences with the applications, and how those varied depending on the target group. The pilot study took place on two events. Participants were less than 15 experts at an international symposium in the field of $5 \mathrm{G}$ technology, three professional dancers, as well as the general public on a public event with more than 50 participants testing the demonstrators.

\subsection{Modern replacement: Vibrotactile feedback for learning dance choreographies}

Concept: In the case of a sudden absence, professional replacement dancers have to be able to learn new choreographies within a short period of time and often separately from the ensemble. In order to raise efficiency, this process has been redefined through user-centred approaches. With the help of a wearable, the dancer receives a haptic feedback that indicates the dance direction. At the same time, there is a floor projection that gives important indicators from the whole ensemble while practicing. Moreover, there is the option to iteratively run through and individualise the learning process using the corresponding 'modern replacement' app.

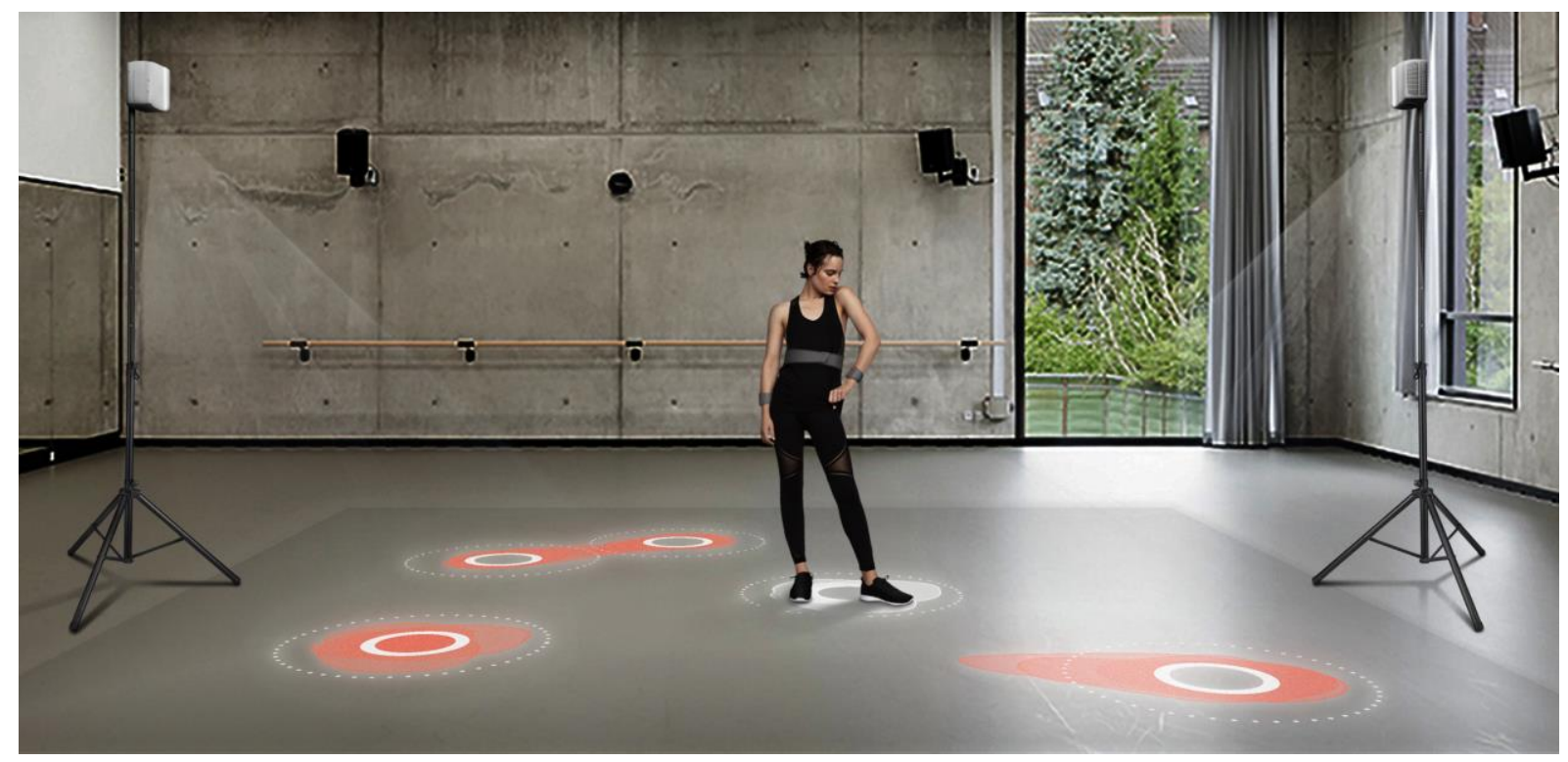

Figure 2. A dancer wears a vibratory belt augmented by a floor projection

Function: The test-demonstrator (as seen in Figure 2) comprises a system made up of a tablet, a vibratory belt, projectors and a monitor with a laptop. All components are connected via a hotspot. The belt has eight integrated vibration motors that can independently give a time-variable vibration feedback. An intuitive User Interface on the tablet enables the interaction with the belt. The monitor displays the particular dance sequence while the projector indicates the corresponding formation of the other dancers. Within the scope of the project, this information has been implemented manually. In 
this way, it was possible to experience the potential of the demonstrator in a 'Wizard-of-Oz' style experiment.

Implementation: The demonstrator was tested at several events, each time with different user groups. An early exhibition at the 5G Summit in Dresden revealed some weaknesses which left considerable room for improvement. General motivation of the visitors to engage with the demonstrator was low. Because the exhibition venue was brightly lit, the floor projection could not be noticed properly. Moreover, the demonstrator was placed in a central position on-stage which raised the inhibition threshold for people to interact with it. Another presumed factor for low engagement was a lack of identification with the application scenario "contemporary dance" among the technology-oriented audience. However, at the Saxony State Price for Design, the demonstrator was displayed and won an award in the category "Young Designers". Taking it along to further school events and the "Long Night of Sciences" event, it was possible to test and evaluate the demonstrator with a broad audience.

After drawing some initial conclusions, the next step was to involve professional dancers in an exemplary sequence to get a more in-depth analysis. The overall motivation and feedback from the next generation demonstrator was very positive. The professional dancers were able to really understand and appreciate the advantages of the new 5G technology. They managed to learn the default sequence in a very short time, profiting from an enhanced sense of space and the directional impulses of the belt.

\subsection{Sensing in motion: Tracking volleyball players for technique adjustments by a digital representation}

Concept: During Volleyball training sessions or in a regular game play situation, it can be difficult to spot and correct small nuances in the technique of a player. With the sensor-suit "Sensing in Motion," it will be possible to track and project one's own motion sequence through a digital twin in real-time. This digital twin can then be mapped with those of professional players with the option to receive direct haptic feedback.

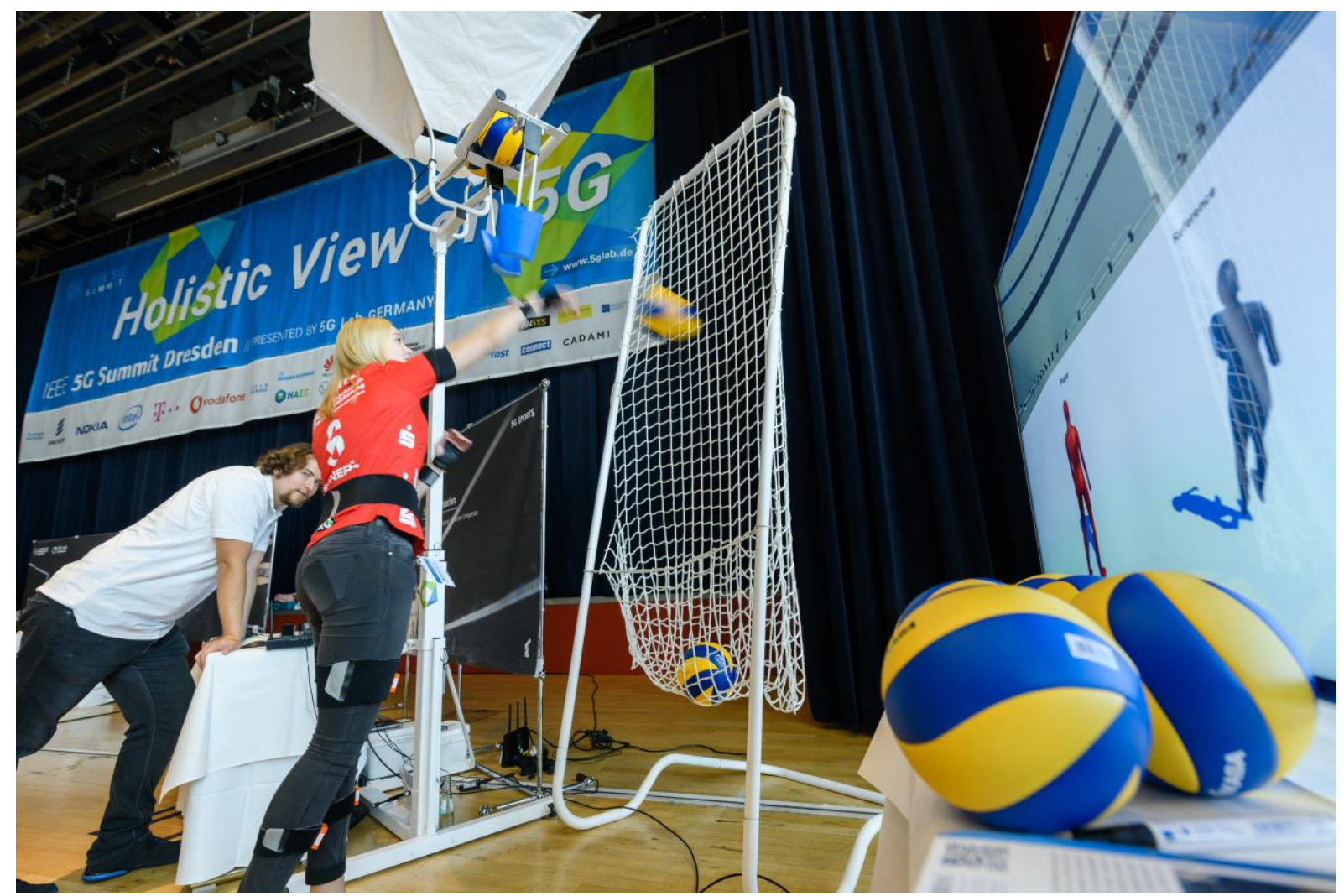

Figure 3. A volleyball player performs a serve simultaneously with the avatar 
Function: The demonstrator, as seen in Figure 3, comprises functional sleeves with integrated sensors that send motion data to the virtual avatar. A monitor is used to display this virtual avatar. Additionally, there is a coach demonstrating the ideal serve technique with the aid of a ball machine.

Implementation: At the trade fair, the demonstrator received a lot of attention. For most attendees, performing a volleyball serve did not present any major difficulties. Another advantage of the demonstrator was its large setup with rather loud sound effects. This led to people being attracted to the display even from larger distances.

\subsection{Lyne Soft ExoSuit: Force feedback for real-time training support and monitoring in rowing}

Concept: The intelligent suit acts as an active training support for professional rowers. It tracks all the relevant motion data of the athlete through integrated sensors. On this data basis, it is possible to affect the musculoskeletal system of the athlete through specialised actuators. The actuators create local forces with the aid of pulling ropes and specifically formed upper- and under-arm sleeves. In order to maintain a consistent oar stroke, the suit provides additional acoustic and haptic signals. The surveillance and adjustment of the settings can take place via visual interfaces such as a tablet or VR device, even over long distances.

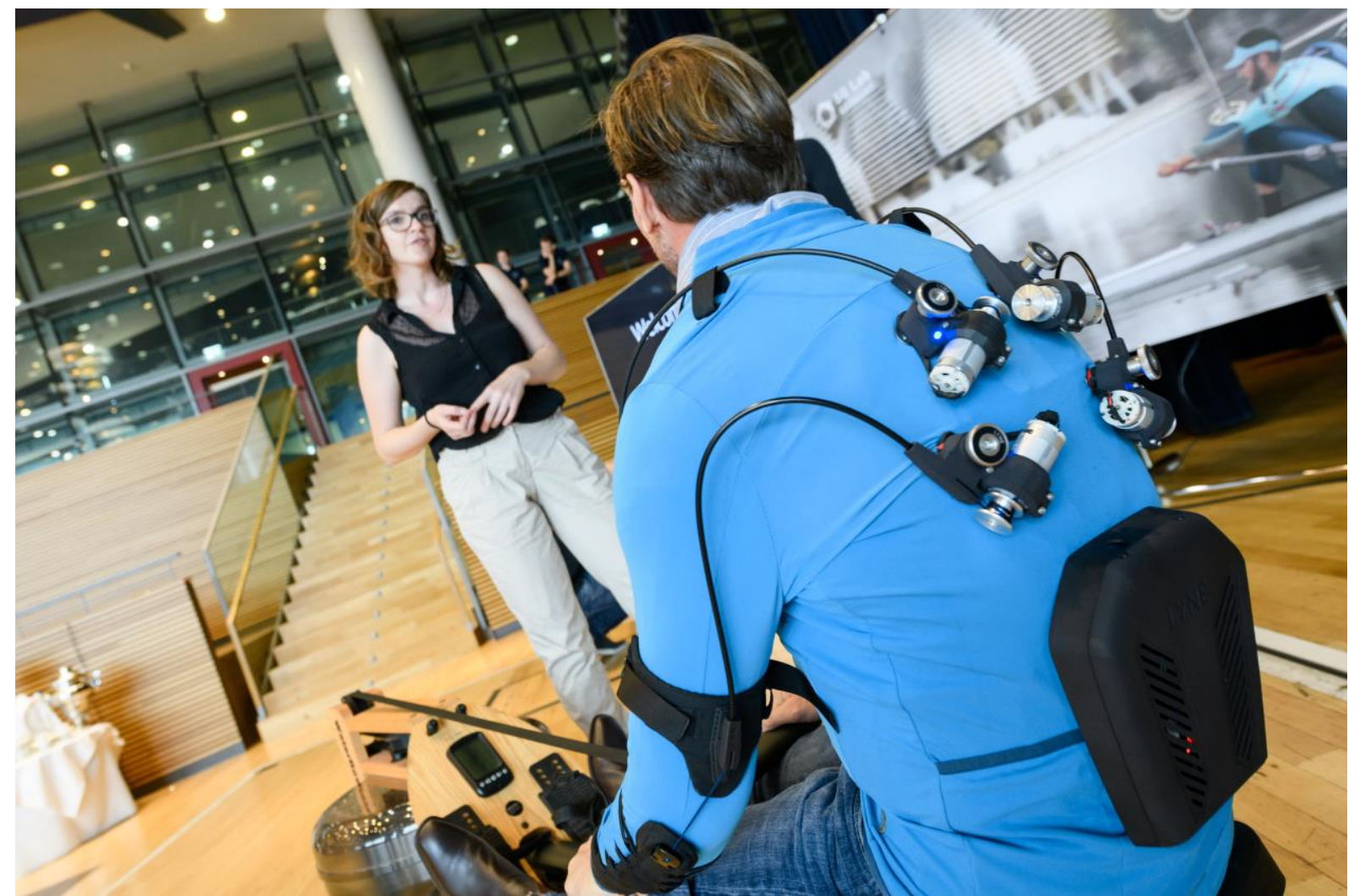

Figure 4. 5G experts tests the soft ExoSuit on a rowing machine

Function: The presentation demonstrator, as seen in Figure 4, is made up of a soft exosuit that can be worn like a jacket. It has six integrated servomotors with cable control. Additionally, there is a batteryand processor-pack to control the motors, situated on the back of the athlete. This battery pack is connected with a user interface that displays the relevant real-time performance data of the athlete on a monitor. In order to be able to test the demonstrator on-site under relatively realistic conditions, it was set up for use with a standard rowing machine.

Implementation: This setup attracted a lot of attention among the attendants of the fair and motivated subjects to further engage with the demonstrator. The servomotors on the suit were emphasised 
through LEDs which led people to make first assumptions about the functionality of the suit. The highlighting of the body-worn technology increased the credibility of the demonstrator.

Negative perceptions of the demonstrator included: a lack of options for customisation, need to tailor it to the user and the weight of the model have been perceived as non-progressive. The expert audience was expecting a lightweight textile system. At the same time, the force of the motors was too low to provoke a noticeable impact. This made it difficult for users to understand the functionality of the demonstrator.

Our observations led to the conclusion that it poses a big challenge to realistically test exoskeletons in an early stage of development. The demands regarding anatomy or motor performance are considerably high, just like the user expectations. This can be due to the popularity of the chosen field of application, or the similarity to established visions from science fiction movies.

Summarised, all three prototypes are body-worn CPS. However, their respective performance and impact on the body vary significantly. "Modern Replacement" is a wearable with vibratory motors that give the user a slight haptic feedback. The sleeves of the "Sensing in Motion" prototype are wearables as well, yet the user receives a purely visual feedback. In contrast, there is the Lyne Soft ExoSuit based on an intensive, reciprocal system of motion control. Drawing comparisons with digital user interfaces, it becomes apparent that information processing and presentation only play a secondary role for body-worn systems. To contribute to the meaningfulness and credibility of such body-worn systems, there's a need for a clear haptic interaction between human and machine. The physical contact with the CPS causes an intense perception of the technology. However, this also entails that errors, such as software bugs or high latency periods, are noticed to a greater extent. Accordingly, the effort to present trustworthy CPS is much higher for body-worn systems than for purely digital prototypes. It is therefore necessary to analyse and identify all relevant factors of influence for the specific fields of application.

\section{Discussion and outlook}

Based to the feedback of the subjects, it can be concluded that the projects succeeded to point out advantages as well as critical aspects of 5G technology. They established a basis for various professional applications that aim to enhance user capabilities. In addition, the demonstrators enabled a dialog about concerned and fears on public events between laypeople and the scientist of 5G technologies. For instance, some people doubted the need for such a technology at all. Those conversations lead to new ideas and approaches for the use of the TaHil technology and consequently the adaption of the demonstrators as well as the use cases.

The tangible demonstrators proved then to be a suitable means to convey the anticipated value of this complex, futuristic technologies and the resulting innovations. Even though not every participant was convinced, it also became apparent that acceptance for new technologies can be raised considerably through examples from popular fields of application, e.g. sports.

According to the St. Gallen Business Innovation Model, we could classify our observations to five key factors regarding the user's perception of the demonstrators as follows:

The performance of the demonstrators in the various application scenarios were assessed differently, also depending on the user's expectations. This plays a bigger role for exoskeletons than for wearables.

Social influence and hedonic motivation turned out to be important factors that can be greatly influenced by the range of demonstrators. In particular, sports use-cases were embraced as exciting and inspiring fields of application. Depending on the interests of the audience, the demonstrators were used to a greater or lesser extent. It is worth mentioning the factor of habit in this regard. Most of the chosen sports applications are activities that are not practiced on a daily basis by the users in the target audience. These are activities that are of particular interest to the users, but don't necessarily have the potential to evoke change regarding their habits. Ultimately, there is always the question of an adequate price-performance ratio. This is where a technology demonstrator should have a plausible answer regarding the respective context and user scenario.

In summary, our approach to, identify valuable factors on the experience of demonstrators is helpful to conduct a more informed design process for future projects. The realisiation quality of the demonstrators 
was overall convincing eventhough the exosuits force feedback was limited. However, the pilot study was insufficiently embedded, consequently, explicit results could not be transferred in our design process. Nevertheless, we could adapt the findings implicitly in the following process of the next demonstrator:

- as target group explicitly laypeople e.g. school students were addressed

- therefore, the factor of hedonic motivation was chosen for communicating TaHil: the design process was enroled in order to design a demonstrator which animates participants to play with the demonstrator "Rock, Paper, Scissors"

- the study is accompanied by adapted evaluation tools of user experience design and social research

However, it has to be clarified in which amount those factors are relevant in case of wearable cyber physical-systems, and if there are even more factors to be considered in the conext of the "Internet of Skills". Futhermore, measuring tools like qualitative and quantitative methods of social and psychology research are required in order to evaluate in more detail, the experience of designed demonstrators representing technological innovations. Generally, with this attempt we expect to develop a design framework for demonstrators targeting their ability to transfer scientific research into meaningful outcomes for society. Therefore, the aim is to motivate through the demonstrators designed as 'translator objects' a shift of the current technology pushed TaHil applications towards design-driven innovations.

\section{Acknowledgement}

Funded by the German Research Foundation (DFG, Deutsche Forschungsgemeinschaft) as part of Germany's Excellence Strategy - EXC 2050/1 - Project ID 390696704 - Cluster of Excellence "Centre for Tactile Internet with Human-in-the-Loop" (CeTI) of Technische Universität Dresden.

\section{References}

Arts, J., Frambach, R. and Bijmolt, T. (2011), "Generalizations on consumer innovation adoption: A metaanalysis on drivers of intention and behavior", Int J Res Marketing, Vol. 28 No. 2, pp. 134-144.

Bäumer, D. et al. (1996), User Interface Prototyping - Concepts, Tools, and Experience, pp. 532-541. https://doi.org/10.1109/ICSE.1996.493447

Bell, F. et al. (2013), "Science fiction prototypes: Visionary technology narratives between futures", Futures, Vol. 50, pp. 15-24. https://doi.org/10.1016/j.futures.2013.04.004

Bendel, O. Wearbales, Springer Gabler, Wiesbaden. https://wirtschaftslexikon.gabler.de/definition/wearables54088/version-368816 Revision von Wearables vom 07.01.2019.

Lauff, C.A., Kotys-Schwartz, D. and Rentschler, M.E. (2018), "What is a Prototype? What are the Roles of Prototypes in Companies?", J. Mech. Des., Vol. 140 No. 6, zuletzt geprüft am 26.01.2020.

Chiesa and Frattini (2011), "Commercializing technological innovation: learning from failures in high-tech markets", J Product Innovation Manag, Vol. 28 No. 4, pp. 437-454.

Dell'Era, C., Marchesi, A. and Verganti, R. (2010), "Mastering technologies in design-driven innovation", Research technology management: RTM, Vol. 53 No. 2, pp. 12-23.

Feiereisen, S., Wong, V. and Broderick, A.J. (2008), “Analogies and mental simulations in learning for really new products: the role of visual attention”, J Product InnovationManag, Vol. 25 No. 6, pp. 593-607.

Garcia, R. and Calantone, R. (2002), "A critical look at technological innovation typology and innovativeness terminology: a literature review", J Product InnovationManag, Vol. 19 No. 2, pp. 110-132.

Gourville, J.T. (2006), "Eager sellers \& stony buyers", Harvard Business Rev, Vol. 84 No. 6, pp. 98-106.

Grimpe, C. and Fier, H.J. (2010). Technol Transf 35: 637. https://doi.org/10.1007/s10961-009-9140-4

Henard, D.H. and Szymanski, D.M. (2001), "Why some new products are more successful than others", $J$ Marketing Res, Vol. 38 No. 3, pp. 362-375.

Kymalainen, T. (2016), "Science Fiction Prototypes as a Method for Discussing Socio-Technical Issues within Emerging Technology Research and Foresight", Athens Journal of Technology \& Engineering.

Lee, E.A. (2006), Cyber-Physical Systems: Are Computing Foundations Adequate?, NSF Workshop on CyberPhysical Systems: Research Motivation, Techniques and Roadmap, Austin, TX.

Moultrie, J. (2015), "Understanding and classifying the role of design demonstrators in scientific exploration", Technovation, Vol. 43-44, pp. 1-16, ISSN 0166-4972, https://doi.org/10.1016/j.technovation.2015.05.002. (http://www.sciencedirect.com/science/article/pii/S0166497215000437) 
Price, R. and Wrigley, C. (2016), "Design and a Deep Customer Insight Approach to Innovation", Journal of International Consumer Marketing, Vol. 28 No. 2, pp. 92-105. https://doi.org/10.1080/08961530.2015. 1092405

Tomczak, T., Vogt, D. and Frischeisen, J. (2016), "How consumers perceive innovation as novelty and meaningfulness as central determinants", In: Hoffmann, C., Lennerts, S., Schmitz, C., Stölzle, W. and Uebernickel, F. (Eds.), Business Innovation: Das St. Galler Modell, Business Innovation Universität St. Gallen.

Utterback, J.M. et al. (2006), Preface: Design-Inspired Innovation, World Scientific Publishing Company.

Venkatesh, V., Thong, J.Y. and Xu, X. (2012), "Consumer acceptance and use of information technology: extending the unified theory of acceptance and use of technology", MIS Quarterly, Vol. 36 No. 1, pp. 157-178.

Verganti, R. (2009), Design Driven Innovation: Changing the Rules of Competition by Radically Innovating What Things Mean, Harvard. 